\title{
Fault Recognition of Underground Cable using Internet of Things
}

\author{
Mukesh Gautam, S. Veena Dhari, Ravitesh Mishra
}

\begin{abstract}
The main objective of this research paper is to propose a system for fault recognition in the cable by utilizing a microcontroller (Arduino). This proposed system will pin down the meticulous location of underground cable by the means of internet, more particularly through the control station in "kilometers". The proposed system's concept is centered on OHM's law i.e. voltage drop may vary including on the span of the fault in cable because of current's variation. In our research we have employed an assortment of resistors to depict the cable's length in "kilometers" and dc voltage is implemented at one of the ends. The fault is discerned by capricious voltage employing an ADC unit (analog-to-digital Converter). The display unit is coupled with the microcontroller for information display about the fault supervening at diverse lines.
\end{abstract}

Keyword: IOT, Cable, microcontroller, Analog to digital converter

\section{INTRODUCTION}

The foremost persistence of the transmission line of the electrical power system is to convey the electrical energy from the electricity generation component to the clients. In this research paper, we incline towards the underground cable in place of overhead for the dissemination since the underground cable is not influenced by any weather circumstances such as lightning, winds, earthquake, frost, earth tremors, high speed, etc. However, once a fault is eventuating at the underground cable, it is a complex modus operandi to perceive whereabouts of the fault to manifest the fault before it upsurges detriment to the power system. So, our goal in this research paper is to ascertain the peculiar position of the fault in the cable. Now the world is becoming digitalized henceforth our project is projected to perceive the situation of the fault in digitalized approach. Nowadays, the underground cable organization is a standard practice that is followed in copious urban regions. The cable's fault may eventuate for any intension, yet the primary issue is to know the unerring location of the cable fault[1].

Fault in every cable is regarded as:

- $\quad$ Any defect and inconsistency.

- Instigated by conductor breakage and insulation

failure

- Dimness or non-homogeneity affecting the enactment of the cable

- Deviation of current from the scheduled path

Revised Manuscript Received on 14 August, 2019.

Dr. MukeshGautam, Department of Computer Science \& Engineering, Rabindranath Tagore University,Bhopal, Madhyapradesh, India.

Dr. S. VeenaDhari, Department of Computer Science \& Engineering, Rabindranath Tagore University,Bhopal, Madhyapradesh, India.

Dr. Ravitesh Mishra,Department of Electronics \& Communication Engineering, Rabindranath Tagore University,Bhopal, Madhyapradesh, India.
Fault in any subterranean cable can be a breakdown in two batches:

Open Circuit Fault: This variety of faults in the subterranean cable occurs because of the failure of one of many conductors. The primary reason for this type of outage incorporates joint failure at places wherein dual cable meets, failure of one or more phases of the circuit breaker. Due to melting of conductor in one of numerous phases. These groupings of faults are also acknowledged as series faults and are better than short-circuit fault as current flowing via cable will cancel out when open-circuit fault takes place.

Short Circuit Fault: Also acknowledged as shunt-faults initiated by the insulation failure among two-phase conductor or among ground plus phase-conductor. It can be further separated into two more classifications namely:

1. Symmetrical Fault: The fault gives escalation to fault that is evacuated by " $120^{\circ}$ " from each other. Also wellknown as balanced-fault arises due to all the three-phases are short-circuited concurrently[2].

2. Unsymmetrical Fault: It is the furthermost common kind of fault that happens in the underground-cable system. This category of outage provides escalation to unsymmetrical fault current that employs unbalancedcurrent in the system.

\section{Fault Location Methods}

It can be majorly classified as:

Online Method: This method employs and enumerates the sampled -voltages along with the current to administrate the fault point location.

Offline Method: In this method, a peculiar unit is employed to scrutinize the underground cable.

There are chiefly two offline methods for fault discerns:

1. Tracer Method: Here the fault-point is discerned by walking along with cable lines. The fault-joint is positioned by directing an audible signal or electromagnetic signal projecting toward the cable. It discerns the fault location very accurately.

2. Terminal Method: It is a method that is employed deprived of tracing to distinguish cable fault position from one or both ends. This technique is employed to discover universal fault area, to accelerate tracing on buried-cable.

\section{SYSTEM METHODOLOGY}

This research paper mostly influenced by the methodology of "OHM's law" where a low DC voltage is applied on the feeder end over a series resistor. The current fluctuates according to the span of the fault ensued in the 
cable. The voltage present across series resistor drops vicissitudes consequently and further passed to an ADC to cultivate precise digital data which is exhibited by the preprogrammed microcontroller in "kilometers". The research work is demonstrated by employing a cluster of resistors denoting cable length in "km's" and fault construction is demonstrated by a set of switches at every single known "kilometer" to crosscheck meticulousness of the same[3].

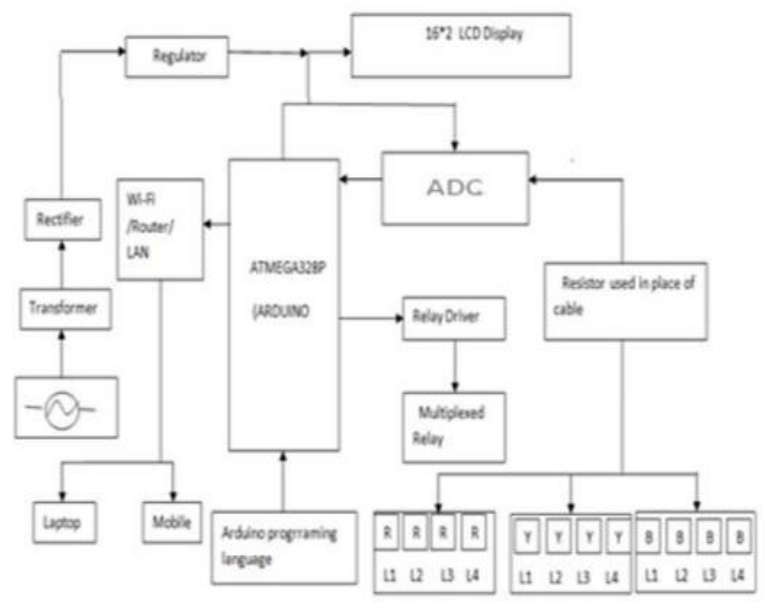

Fig. 1 underground cable is constructed on Arduino, including 4 bridge rectifier, converter and regulator $\}$, cable part, controlling part, display part". The transformer employed here is basically a step-down transformer. The voltage from the transformer is applied to the bridge-rectifier that further transfigures AC signal to DC signal and we have employed a regulator for the production of constant DC voltage. We have employed a group of resistors organized with switches in the cable part. The current intuiting fragment is employed as fault originators to point toward the fault location. The current intuiting fragment senses the abruption in current by ascertaining the voltage-drop[4]. is associated with an analog to digital converter that obtains input signal from the current sensing circuit and notifies this input signal into a digital signal and transmissions it to Arduino. As a fragment of the controlling unit, Arduino amends essential analysis related to the distance of the fault that occurred in the underground cable. The Arduino also energies a relay-driver contrivance which controls the switching operation of a set of relays for appropriate assembly of the cable at every phase. The display unit encompasses of LCD display associated with the Arduino for screening the status of the working condition of cable of every phase and cable distance at a specific phase, in case of any fault[5].

\section{Power Supply}

The power supply is an electronics-equipment that transmits the electrical energy to a load. The most important function of the power supply is to modify the electrical energy from one form to another as a consequence power supplies are so often called electric power converters. A
The anticipated system of fault distance locator in the parts "DC power supply $\{230 \mathrm{v}$ steps down transformer,

The following step encompasses of a controlling-part that

direct current power supply supplies a constant surge of DC voltage to its load. Depending on the system, a DC power supply can be powered from a DC or AC source like power mains[6].

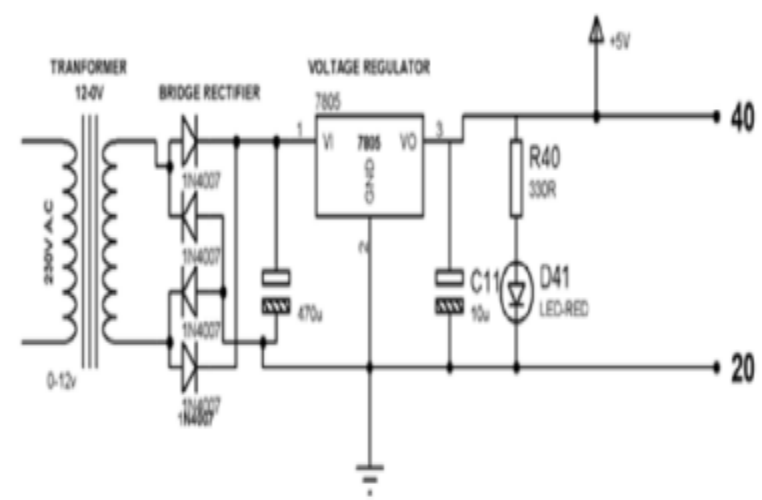

Fig. 2

\section{Transformer}

It is an apparatus that supports during electrical energy transmission from one circuit to another circuit with the help of a magnetic field medium deprived of alteration in the frequency. The transformer is also employed to upsurge or for the diminution of voltage that is flowing via a transmission line. It employs a "magnetic induction principle". It encompasses, in its humblest form, of two or more coils of insulated wire twisted on a laminated steel core. When voltage is passed through first coil, known as primary, it magnetizes the iron core. A voltage is then generated within the second coil, referred to as the secondary (or output coil). The variation of voltage (or voltage ratio) among the coils be determined by the turn's ratio of both of the coils[7].

\section{Rectifier}

The bridge rectifier is employed for converting an ac voltage into dc voltage by "means of both positive and negative half cycles of the input ac voltage". The alternating current voltage is implemented to the diagonally opposite ends of the bridge. The load resistance is involved among the other two ends of the bridge.

\section{RESULT AND CONCLUSION}

In the current system, we have detected the precise location of the fault in the underground cable from the feeder end in kilometer by the implementation of the Arduino (ATMEGA328P). We have employed OHM's law principle so that fault can be straightforwardly detected and repaired.

In the proposed system, we have noticed that the accurate position of fault in the cable underneath the surface from the end of a feeder in "kilometer" along with utilization of Arduino "(ATMEGA328P)". In this research paper we have also employed "OHM's law" principle to detect the fault in the cable easily for reparation. 
We have two cases

Case 1: When there no-fault

\section{Underground Cable Fault Detector}

LINE 1
LINE 2
IOK

Case 1 ( If there is no fault)

Case 2: When there a fault at line 1

Underground Cable Fault Detector

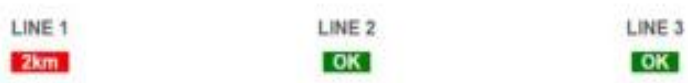

Case 2 ( if there is a fault at line 1 within $2 \mathrm{~km}$ )

Case 3: When there a fault at line 1

Underground Cable Fault Detector

$\begin{array}{lll}\text { LINE 1 LINE 2 } & \text { LINE 3 } \\ \text { I2km } & \text { I2km }\end{array}$

Case 3 (if there is fault at all three cable within $2 \mathrm{~km}$ )

\section{FUTURE SCOPE}

In this paper, only the short circuit error position has been established, but we can also predict open circuit errors for future leveraging an ac-circuit condenser for the measurement of impedance adjustments and the estimation of fault distances.

\section{REFERENCES}

1. T. S. Sidhu and Z. Xu, "Detection of incipient faults in distribution underground cables," IEEE Trans. Power Deliv., 2010.

2. B. Kasztenny, I. Voloh, and C. G. Jones, "Detection of incipient faults in underground medium voltage cables," in 2009 Power Systems Conference: Advance Metering, Protection, Control, Communication, and Distributed Resources, PSC 2009, 2009.

3. J. Yang, J. E. Fletcher, and J. O'Reilly, "Short-circuit and ground fault analyses and location in VSC-based DC network cables," IEEE Trans. Ind. Electron., 2012.

4. M. Gilany, D. K. Ibrahim, and E. S. Tag Eldin, "Traveling-wave-based fault-location scheme for multiend-aged underground cable system," IEEE Trans. Power Deliv., 2007.

5. K. Y. Parikh, B. Kumar, and V. Raval, "GSM Based Underground Cable Fault Distance Locator,” 2018.

6. X. Yang, M. S. Choi, S. J. Lee, C. W. Ten, and S. Il Lim, "Fault location for underground power cable using distributed parameter approach," IEEE Trans. Power Syst., 2008.

7. C. W. Mclyman, Transformer and Inductor Design Handbook. 2010. 